\title{
The Antagonist of Retinoic Acid Receptor $\alpha$, ER- 5089I Antagonizes the Inhibitive Effect of All-Trans Retinoic Acid and Rescues Bone Morphogenetic Protein 2-Induced Osteoblastogenic Differentiation
}

This article was published in the following Dove Press journal:

Drug Design, Development and Therapy

\begin{abstract}
Siqian Wang, ${ }^{1, *}$ Wenjuan $\mathrm{Bi}^{2, *}$ Yi Liu, ${ }^{3, *}$ Jiayi Cheng, (D) ${ }^{4}$ Wei Sun, ${ }^{5}$ Gang Wu, (iD) ${ }^{4}$ in Xu'

'Department of Implantology, School and Hospital of Stomatology, Shandong University \& Shandong Key Laboratory of Oral Tissue Regeneration \& Shandong Engineering Laboratory for Dental Materials and Oral Tissue Regeneration, Jinan, Shandong Province, People's Republic of China; ${ }^{2}$ College of Stomatology, North China University of Science and Technology, Tangshan, Hebei Province, People's Republic of China; ${ }^{3} \mathrm{Key}$ Laboratory of Oral Medicine, Guangzhou Institute of Oral Disease, Stomatology Hospital of Guangzhou Medical University, Guangzhou Medical University, Guangzhou, People's Republic of China; ${ }^{4}$ Department of Oral Implantology and Prosthetic Dentistry, Academic Centre for Dentistry Amsterdam (ACTA), University of Amsterdam (UvA) and Vrije Universiteit Amsterdam (VU), Amsterdam, The Netherlands; ${ }^{5}$ The Affiliated Stomatology Hospital, Zhejiang University School of Medicine, Hangzhou, Zhejiang Province, People's Republic of China

*These authors contributed equally to this work
\end{abstract}

Correspondence: Gang Wu

Department of Oral Implantology and Prosthetic Dentistry, Academic Centre for Dentistry Amsterdam (ACTA), University of Amsterdam (UvA), Gustav Mahlerlaan 3004 , Amsterdam 108ILA, The Netherlands

Tel +3I 205980866

Fax +31 205980333

Email g.wu@acta.nl

Xin Xu

Department of Implantology, School and Hospital of Stomatology, Shandong University \& Shandong Key Laboratory of Oral Tissue Regeneration \& Shandong Engineering Laboratory for Dental Materials and Oral Tissue Regeneration, No. 44-I, Wenhua Xi Road, Jinan, Shandong 2500I2, People's

Republic of China

Tel/Fax +86-53I-88382923

Email xinxu@sdu.edu.cn
Background: Hypervitaminosis A, alcoholism or medical treatment for acute promyelocytic leukaemia may cause unphysiologically high accumulation of all-trans retinoic acid (ATRA), which could inhibit osteoblastogenesis, thereby triggering osteoporosis. We have shown that bone morphogenetic protein-2 (BMP-2) can only partially antagonize the inhibitive effects of ATRA. In this study, we hypothesized that antagonists of retinoic acid receptors (RARs) could further antagonize the inhibitive effect of ATRA and rescue BMP2-induced osteoblastogenesis. Materials and Methods: We first screened the dose-dependent effects of the specific antagonists of RAR $\alpha, \beta$ and $\gamma$ and transforming growth factor-beta receptor (ER-50891, LE-135, MM11253, and SB-43142, respectively) on ATRA-induced inhibition of the total cell metabolic activity and proliferation of preosteoblasts. We selected ER-50891 and tested its effects on osteoblastogenesis with the presence or absence of $1 \mu \mathrm{M}$ ATRA and/or $200 \mathrm{ng} / \mathrm{mL}$ BMP-2. We measured the following parameters: Alkaline phosphatase activity (ALP), osteocalcin (OCN) expression and extracellular matrix mineralization as well as the level of phosphorylated Smad1/5. Results: ER-50891 but not LE-135, MM11253, or SB-431542 significantly antagonized the inhibition of ATRA and enhanced the total cell metabolic activity and proliferation of preosteoblasts. Dose-dependent assays show ER-50891 could also rescue ATRA inhibited OCN expression and mineralization with or without the induction of BMP. ER-50891 also suppressed the ALP activity that was synergistically enhanced by BMP and ATRA. Neither ATRA, nor ER-50891 or their combination significantly affected the level of BMP-induced phosphorylated Smad1/5.

Conclusion: The antagonist of RAR $\alpha$, ER-50891 could significantly attenuate ATRA's inhibitive effects on BMP 2-induced osteoblastogenesis.

Keywords: bone morphogenetic protein 2, all-trans retinoic acid, retinoic acid receptor, osteoblastogenesis, transforming growth factor beta

\section{Introduction}

Bone tissues with sufficient quantity and quality are highly important for the proper functions of musculoskeletal systems and therein-implanted medical devices, such as dental implants. ${ }^{1}$ As a paramount biological process to maintain bone tissue and repair bone defects, mesenchymal stem cells are osteogenically committed to become a preosteoblast and thereafter undergo osteoblastogenesis. ${ }^{2}$ Osteoblastogenesis comprises a series of sequential cellular events, such as proliferation, alkaline phosphatase (ALP) expression (early differentiation marker), osteocalcin (OCN) expression (late 
differentiation marker) and final extracellular matrix mineralization. ${ }^{3}$ In pathogenic conditions, osteoblastogenesis can be inhibited by metabolites or drugs, which may result in various bone diseases, such as osteoporosis ${ }^{4}-$ a metabolic bone disease characterized by significantly reduced density and deteriorated microstructure of bone tissue with increased risks of fractures. ${ }^{5}$

One of such metabolites or drugs is all-trans retinoic acid (ATRA). ${ }^{6}$ In physiological microenvironments, ATRA is a metabolite of alcohol and vitamin A and widely involved in regulating a large variety of physiological events, such as epithelial differentiation, ${ }^{7}$ breast cancer ${ }^{8}$ and embryogenic development. ${ }^{9}$ Unhealthy dietary habits such as hypervitaminosis A can cause the unphysiological accumulation of ATRA in human body, which may result in a series of diseases, such as neural toxicity and osteoporosis. ${ }^{10-12}$ On the other hand, ATRA may also, at least partially, mediate the detrimental effects of alcohol abuse. ${ }^{13}$ Alcoholism is highly prevalent worldwide with a prevalence of $18.4 \%$ adult for heavy alcohol abuse. ${ }^{14}$ Chronic alcohol abuse can result in low bone density, ${ }^{15-18}$ bone fragility and fractures. ${ }^{15,19-21}$ Data from animal studies show that alcohol abuse is associated with significantly reduced osteogenesis ${ }^{22}$ and delayed implant osteointegration, ${ }^{23}$ which is at least partially, due to the significantly reduced osteoblastogenesis. ${ }^{24}$ Alcoholism can result in compromised osteoinduction, leading to compromised bone defect healing. ${ }^{24}$ Furthermore, prenatal alcohol exposure also significantly affects fetal bone development. ${ }^{25}$ Apart from these dietary aspects, high-dose ATRA is also given to adult patients to treat acute promyelocytic leukemia (APL) ${ }^{26}$ For this purpose, oral administration of high dosage $\left(45 \mathrm{mg} / \mathrm{m}^{2}\right)$ of ATRA is conventionally recommended, which results in a median concentration of approximately $1 \mu \mathrm{M}$ in plasma. ${ }^{27,28}$ Osteoporosis occurs as a side effect of ATRA. ${ }^{29}$ ATRA at pharmacological concentration of $1 \mu \mathrm{M}$ is frequently used in in-vitro experiment. ${ }^{30}$ All these findings suggest that ATRA has an inhibitive effect on osteoblastogenesis. ATRA takes effect through two types of nuclear receptors, e.g. retinoic acid receptors (RARs) and retinoid X receptors (RXRs). ${ }^{10}$ Each type of receptors is comprised of three subtypes $(\alpha, \beta$, and $\gamma$ ). The RARs can bind RXRs to form heterodimers that directly modulate target gene expression through retinoic acid response elements (RAREs). ${ }^{31}$ Apart from RAR-mediated signaling, ATRA is also reported to inhibit cell proliferation by inducing endogenous transforming growth factor $\beta$ s (TGF$\beta \mathrm{s}) .{ }^{32}$ TGF- $\beta$ s bind to TGF- $\beta$ receptors and cause cell cycle arrest. ${ }^{32-34}$ Hitherto, it is unclear which receptor plays a critical role in the inhibitive effect of ATRA on osteoblastogenesis.
On the other hand, in clinic, bone morphogenetic proteins (BMPs), one group of highly potent osteoinductive growth factors, are widely used to promote osteoblastogenesis so as to facilitate rapid and complete bone healing. BMP-2 or BMP7 carried by absorbable collagen sponge, have been approved by FDA to apply in clinic aiming to promote bone regeneration. ${ }^{35,36}$ BMPs take effects by binding to BMP receptor complex on cell membrane, which results in significantly enhanced level phosphorylated Smad1/5 (p-Smad1/5). By forming a complex with Smad4, P-Smad1/5 translocates into nucleus and binds to DNA promoters so as to induce the expression of osteogenic genes, leading to osteoblastogenesis. Recently, we report that $1 \mu \mathrm{M}$ ATRA inhibits osteoblastogenesis of both bone marrow stromal cells and mouse calvarial preosteoblast cell (MC3T3-E1 cell line) ${ }^{37-39}$ We also show that ATRA can antagonize the promoting effects of various BMPs on the expression of key osteogenic genes (such as Runx2, Osterix, Dlx5 and Smad1) irrespective of the BMPs' potencies. ${ }^{39}$ However, hitherto, it is unclear which receptor plays a critical role in the inhibition of ATRA on BMP2-induced osteoblastogenesis.

In this study, we adopted specific antagonists to different RARs and also TGF- $\beta$ type I receptor kinase with an aim to assess the roles of different receptors in the inhibitive effects of ATRA on BMP-2-induced osteoblastogenesis.

\section{Materials and Methods Cell Culture}

A preosteoblast cell line (MC3T3-E1, subclone 4, CRL-2593) was purchased from the American Type Culture Collection (ATCC). Cells were cultured in growth medium consisting of $\alpha$-MEM with $10 \%$ (v/v) FBS (fetal bovine serum) (Gibco Invitrogen, Grand Island, NY), $100 \mathrm{U} / \mathrm{mL}$ streptomycin and penicillin at $37^{\circ} \mathrm{C}$ and $5 \% \mathrm{CO}_{2}$ in a humidified atmosphere. Media change was performed every 3 days. When $80 \%$ confluency was reached, cells were plated at a final concentration of at $5 \times 10^{3}$ cells/well in 24-well plates for measuring total cell metabolic activity and DNA content; for at $2.5 \times 10^{4}$ cells/well in 48-well plates for measuring ALP activity, total protein, OCN and extracellular matrix mineralization (alizarin red staining); or $1 \times 10^{4}$ cells/well in 96-well plates for measuring p-Smad1/5 using cell-based enzyme-linked immunosorbent assay (ELISA). Except for extracellular matrix mineralization, cells were seeded in plates in a low-serum medium ( $2 \%$ FBS); $24 \mathrm{hrs}$ after seeding, cells were first starved in the low-serum media ( $0.5 \%$ FBS) for another $24 \mathrm{hrs}$ and then followed by drug treatment also in media containing $0.5 \%$ FBS. 


\section{Treatment of Preosteoblasts}

ATRA was purchased from Sigma-Aldrich. All the receptor antagonists and BMP-2 (CHO derived) were purchased from R\&D Systems, Inc. Cultured preosteoblasts were treated with RAR $\alpha$ antagonist ER-50891, RAR $\beta$ antagonist LE135, RAR $\gamma$ antagonist MM-11253 $(0,1,2$ and $3(\mu \mathrm{M}))$, or TGF-beta antagonist SB-431542 $(0,3,6$ and $9(\mu \mathrm{M}))$, in the absence and presence of $1 \mu \mathrm{M}$ ATRA, during the entire period of differentiation of MC3T3-E1, respectively. For further studies, cells were treated with $200 \mathrm{ng} / \mathrm{mL}$ BMP-2.

\section{Screening Receptor Antagonists}

The parameters of total cell metabolic activity and proliferation (DNA content) were measured in order to identify which specific receptor ATRA ligand binds to. After $24 \mathrm{hrs}$ starving culture, the cells were treated with ATRA or antagonist for 3 days. The results show that the ER-50891, RAR-alpha antagonist group had a significant difference in total cell metabolic activity and DNA content. Instead, the other receptor antagonists LE-135, MM-11253, SB-431542 made no significant difference when comparing with the ATRA group, respectively, with a similar effect level. As a consequence, the receptor in this study was determined as RAR-alpha.

\section{ER-5089| Concentration Selection Test}

Furthermore, to assess the concentration range for how RARalpha antagonist ER-50891 involves in the ATRA signaling cascade, a series of dose-response tests on cell metabolic activity, DNA content, ALP activity, total protein expression, and osteocalcin release were performed as described in the following sections 4 days post-treatment. ER-50891, RARalpha antagonist group on the concentration of $0.25 \mu \mathrm{M}, 0.5$ $\mu \mathrm{M}, 1 \mu \mathrm{M}, 1.5 \mu \mathrm{M}, 2 \mu \mathrm{M}$ and $3 \mu \mathrm{M}$ significantly decreased the ALP activity. These results showed that the ER-50891 antagonized the enhancing effect of ATRA on ALP activity and decreased ALP activity to the similar level as control when 2-3 MM ER-50891 was applied. Besides, ER-50891, RARalpha antagonist group has a significant increase in cell proliferation and osteocalcin release. Consequently, the concentrations in this study were determined at $1.5 \mu \mathrm{M}$ and $3 \mu \mathrm{M}$.

\section{Total Cell Metabolic Activity and Proliferation}

To analyze the effect of ATRA on MC3T3-E1 proliferation in response to various concentrations of different RAR antagonists ER-50891, LE-135, MM-11253, and TGF-beta antagonist SB-431542, the total cell metabolic activity in the absence or presence of $1 \mu \mathrm{M}$ ATRA was gauged using the alamar Blue ${ }^{\mathrm{TM}}$ cell viability assay (Invitrogen Corporation, Carlsbad, CA, USA) following the manufacturer's instruction. In short, after a 3-day treatment, the alamar Blue ${ }^{\mathrm{TM}}$ reagent was added into the growth medium for an incubation of $4 \mathrm{hrs}$. After the incubation period, fluorescence intensity, was measured at EX 540 nm/EM 590 nm using SpectraMax M5 Molecular Devices (Sunnyvale, CA, USA). Proliferation of cells were measured using a DNA quantification assay. The QuantiT ${ }^{\mathrm{TM}}$ PicoGreen dsDNA Assay kit (Invitrogen, OR, USA) was used according to the instruction. The fluorescent intensity of each sample was measured using SpectraMax M5 Molecular Devices as well with a setting of EX480 nm/EM520 nm.

\section{ALP Activity and Total Protein Expression}

After a 3-day treatment, ALP activity and protein expression were measured using para-Nitrophenylphosphate as a substrate using LabAssay ${ }^{\mathrm{TM}}$ ALP colorimetric assay kit (Wako Pure Chemicals, Osaka, Japan) according to the manufacturer's protocol. Briefly, $10 \mu \mathrm{L}$ aliquots of cell lysates were added to 96 -well plates. Thereafter, $50 \mu \mathrm{L}$ of 2 -amino2-methyl-propanol buffer ( $\mathrm{pH} 10.3$ ) and $50 \mu \mathrm{L}$ of $15.2 \mathrm{mM}$ paranitrophenylphosphate (PNPP) in a $2 \mathrm{mM}$ solution of magnesium chloride were added and incubated at $37^{\circ} \mathrm{C}$ for 1 hrs. The reaction was stopped by adding $200 \mu \mathrm{L}$ stop solution (1 $\mathrm{N}$ solution of sodium hydroxide). The amount of produced paranitrophenol was gauged spectrophotometrically at $405 \mathrm{~nm}$ using the plate reader SYNERGYMx $\left(\right.$ BioTek $^{\circledR}$, Winooski, VT) with Gen 51.09 software. The total protein content was measured using a BCA Protein Assay kit (Beyotime, China). Results are expressed as folds of ALP activity. ALP activity was calculated by dividing the amount of paranitrophenol by protein content. Results were expressed by calculating the fold changes in comparison with the control.

\section{Osteocalcin Expression}

Osteocalcin (OCN), referred to as a late marker of osteoblastic differentiation, was analyzed using an enzyme-linked immunosorbent assay (ELISA) for mouse OCN (EIA kit, Biomedical Technologies, Stoughton, MA, USA) according to the manufacturer's protocol. After a 3-day treatment, supernatants of cell culture were collected and centrifuged (20,000 rpm, $\left.4^{\circ} \mathrm{C}, 15 \mathrm{~min}\right)$ before analysis. The absorbance was measured at $450 \mathrm{~nm}$. Results are expressed as the fold changes of OCN in comparison with control. 


\section{Extracellular Matrix Mineralization}

We compared the area of mineralized nodules in samples stimulated by $200 \mathrm{ng} / \mathrm{mL}$ BMP-2 in the absence or presence of $1 \mu \mathrm{M}$ ATRA and ER-50891 (1.5 $\mu \mathrm{M}$ and $3 \mu \mathrm{M})$. The mineralized nodules formation was analyzed using Alizarin red staining at the time points of 19 days after induction. Quadruplicate cell cultures were prepared and then treated with an osteogenic medium (10\% FBS, $50 \mu \mathrm{g} / \mathrm{mL}$ L-ascorbic acid, and 10mM beta-glycerophosphate; SigmaAldrich, St. Louis, MO, USA) containing 200 ng/mL BMP2 plus $1 \mu \mathrm{M}$ ATRA, as well as $1.5 \mu \mathrm{M}$ or $3 \mu \mathrm{M}$ ER-50891. The medium was replaced every 3 days. On Day 19, extracellular matrix mineralization was determined by alizarin red staining at pH 4.2 (Sigma-Aldrich, St. Louis, MO, USA). Each well was photographed using NIS-Elements F2.20 (Nikon Eclipse $80 \mathrm{i}$, Tokyo, Japan), and the mineralized areas were then quantified using Image-Pro Plus 6.0 software.

\section{Cell-Based ELISA}

After a 3-day treatment, P-Smad1/5 was measured using a cell-based ELISA kit (\#KCB7660, R\&D Systems, Inc., Minneapolis, MN, USA) kit and compared to glyceraldehyde 3-phosphate dehydrogenase (GAPDH) in the nuclear according to the instructions.

\section{Statistical Analysis}

All results are shown as means \pm standard deviations (SD). Individual experiments were performed at least three times. The data were analyzed using the GraphPad Prism 6 version 6.0 (GraphPad Software Inc, La Jolla, CA). For comparison among groups, one-way analysis of variance (ANOVA) was conducted. For comparison between two groups, unpaired $t$-test was conducted. $p<0.05$, was considered a statistically significant difference.

\section{Results}

\section{ATRA Inhibited the Total Cell Metabolic} Activity and Proliferation of Preosteoblasts, Which Could Be Rescued by the Antagonist of RAR $\alpha$

We first evaluated the influence of the antagonists on the total cell metabolic activity and proliferation of preosteoblasts. $1 \mu \mathrm{M}$ ATRA caused almost $50 \%$ reduction of total cell metabolic activity compared to the control group. The 1 $\mu \mathrm{M}$ or $2 \mu \mathrm{M} \mathrm{RAR} \alpha$ antagonist ER-50891 alone significantly promoted the total cell metabolic activity of pre-osteoblasts
(Figure 1A). In contrast, RAR $\beta$ antagonist LE-135 alone at all concentrations resulted in significantly lower total cell metabolic activity (Figure 1B). Neither RAR $\gamma$ nor TGF- $\beta$ antagonists alone could significantly influence the total cell metabolic activity of preosteoblasts (Figure 1C and D). The inhibitive effect of ATRA could be relieved only by the antagonist of RAR $\alpha$ (Figure 1A) but neither RAR $\beta$ nor RAR $\gamma$ nor TGF- $\beta$ (Figure 1B-D). A very similar pattern was also found in the effects of the antagonists on the DNA content (Figure 2-D). ATRA significantly inhibited the DNA content. Antagonist of $\operatorname{RAR} \alpha$ but neither RAR $\beta$ nor RAR $\gamma$ nor TGF- $\beta$ significantly antagonized the inhibitive effects of ATRA on the DNA content of preosteoblasts. Since the total cell metabolic activity and proliferation of osteogenic cells are highly important for osteogenesis and only RAR $\alpha$ antagonist ER-50891 could antagonize the inhibitive effects of ATRA, we adopted ER-50891 in the following experiments.

\section{ER-5089I Dose-Dependently Antagonized the Promoting Effect of ATRA on ALP Activity and Also Its Inhibitive Effects on Cell Viability, Proliferation, Total Protein and OCN Expression}

We performed dose-dependent assay to identify the antagonistic effect of ER-50891 on the effects of ATRA. From 0.5 to $1.5 \mu \mathrm{M}$, ER-50891 significantly antagonized the inhibitive effect of ATRA and dose-dependently increased the total cell metabolic activity of preosteoblasts (Supplementary Figure 1A). In the concentration range of 1-3 $\mu \mathrm{M}$, the total cell metabolic activity was elevated to a similar $(1 \mu \mathrm{M}$ and $3 \mu \mathrm{M})$ or significantly higher level $(1.5 \mu \mathrm{M}$ and $2 \mu \mathrm{M})$ in comparison with the control (Supplementary Figure 1A). ER-50891 of all the selected concentrations resulted in a significantly higher level of DNA content than the group of ATRA alone, which reached a similar level as the control (Supplementary Figure 1B). ATRA decreased total protein content to $48.0 \%$ of the control group. In contrast, ER-50891 dose-dependently increase the total protein content of preosteoblasts (Figure $3 \mathrm{~A}$ ) with protein content recovered to $80.6 \%$ of the control group. ATRA increased ALP activity by 2.5 fold, which could be dose-dependently inhibited by ER50891 (Figure 3B). ATRA decreased OCN expression to $23.4 \%$ of the control, which could be dose-dependently 

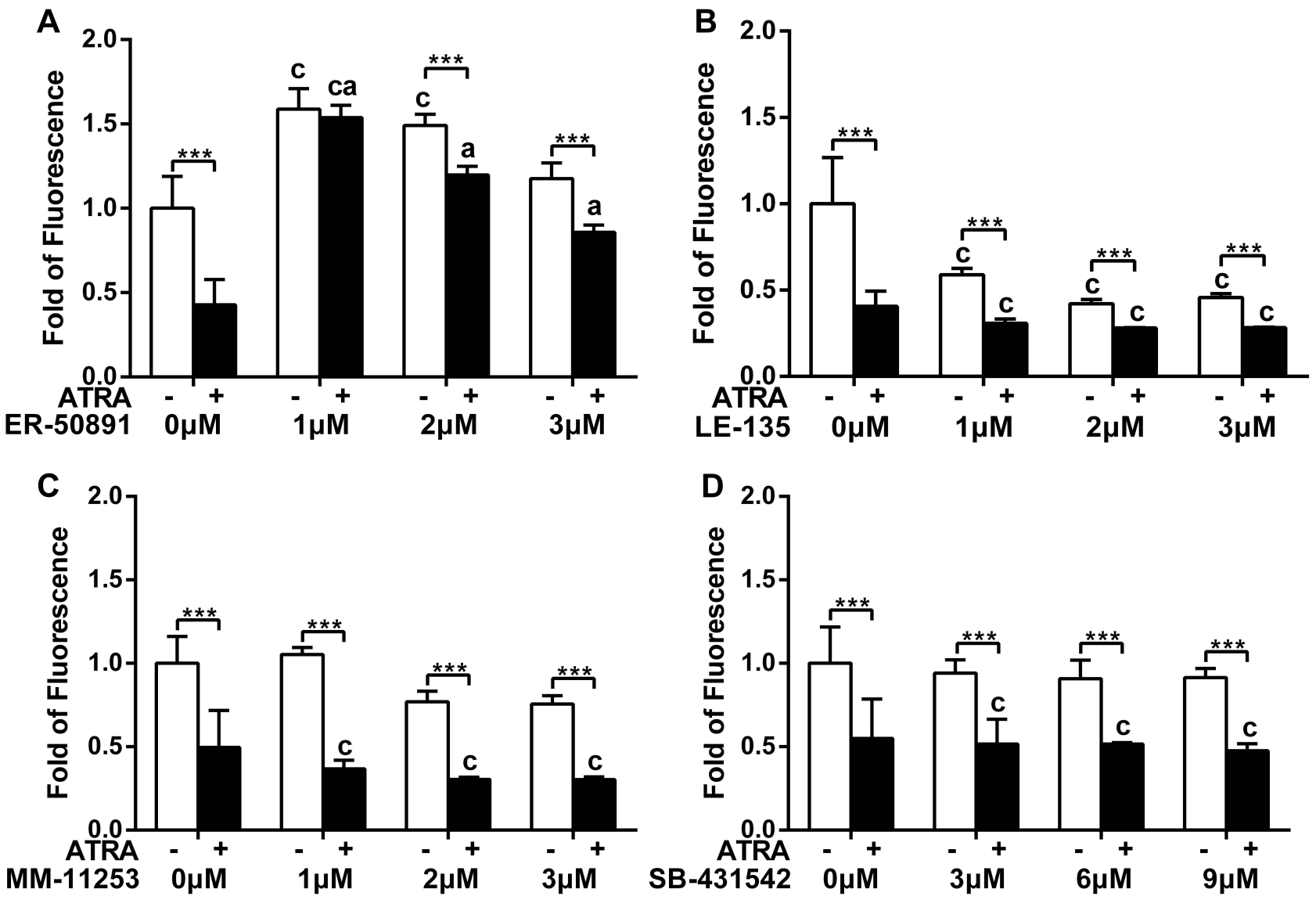

\section{$(-) 1 \mu M$ ATRA a:compared with ATRA group, $p<0.05$ $c:$ compared with control group, $p<0.05$}

Figure I Fold changes of total cell metabolic activity of murine calvarial pre-osteoblasts (MC3T3-EI cells) treated with or without I $\mu$ M ATRA in the presence or absence of different receptor antagonists. RAR-alpha antagonist ER-5089I (A), RAR-beta antagonist LE-I35 (B), RAR-gamma antagonist MM-II253 (C), TGF-beta antagonist SB431542 (D) were adopted. All data are presented as mean values together with the standard deviation. $c$ : significant difference $(P<0.05)$ in comparison with the control group. a: significant difference $(p<0.05)$ in comparison with the ATRA (alone) group. ${ }^{*} * * 00.001$.

relieved by ER-50891. ER-50891 at 2 and $3 \mu \mathrm{M}$ almost completely rescued the OCN expression (Figure 3C).

\section{ATRA Promoted ALP Activity and Inhibited OCN Expression Induced by BMP-2, Which Could Be Antagonized by ER-5089I}

ATRA or $200 \mathrm{ng} / \mathrm{mL}$ BMP-2 alone could promote ALP activity by 2.1 and 1.9 folds, respectively, while ER-50891 alone did not significantly influence ALP activity (Figure 4A). ATRA and BMP-2 synergistically promoted ALP activity by 18.9 folds in preosteoblasts, which could be significantly inhibited by ER 50891. BMP-2 significantly enhanced OCN by 5.9 folds in comparison with the control, which was not influenced by ER-50891. ATRA could significantly suppress OCN expression by $84.2 \%$, which could be significantly relieved to $41.1 \%$ of the control group by ER-50891 (Figure 4B). ATRA also inhibited BMP-2-induced OCN expression by $90.0 \%$. ER-50891 could antagonize such an effect of ATRA, but only rescue the OCN expression to a similar level as the control (Figure 4B).

\section{ER-5089I Attenuated the Inhibitive} Effects of ATRA on BMP-2-Induced

\section{Extracellular Matrix Mineralization}

Nineteen days after treatment, treatment with BMP-2 significantly promoted extracellular matrix mineralization than the control (no ATRA, no ER-50891; Figure 5A). In 

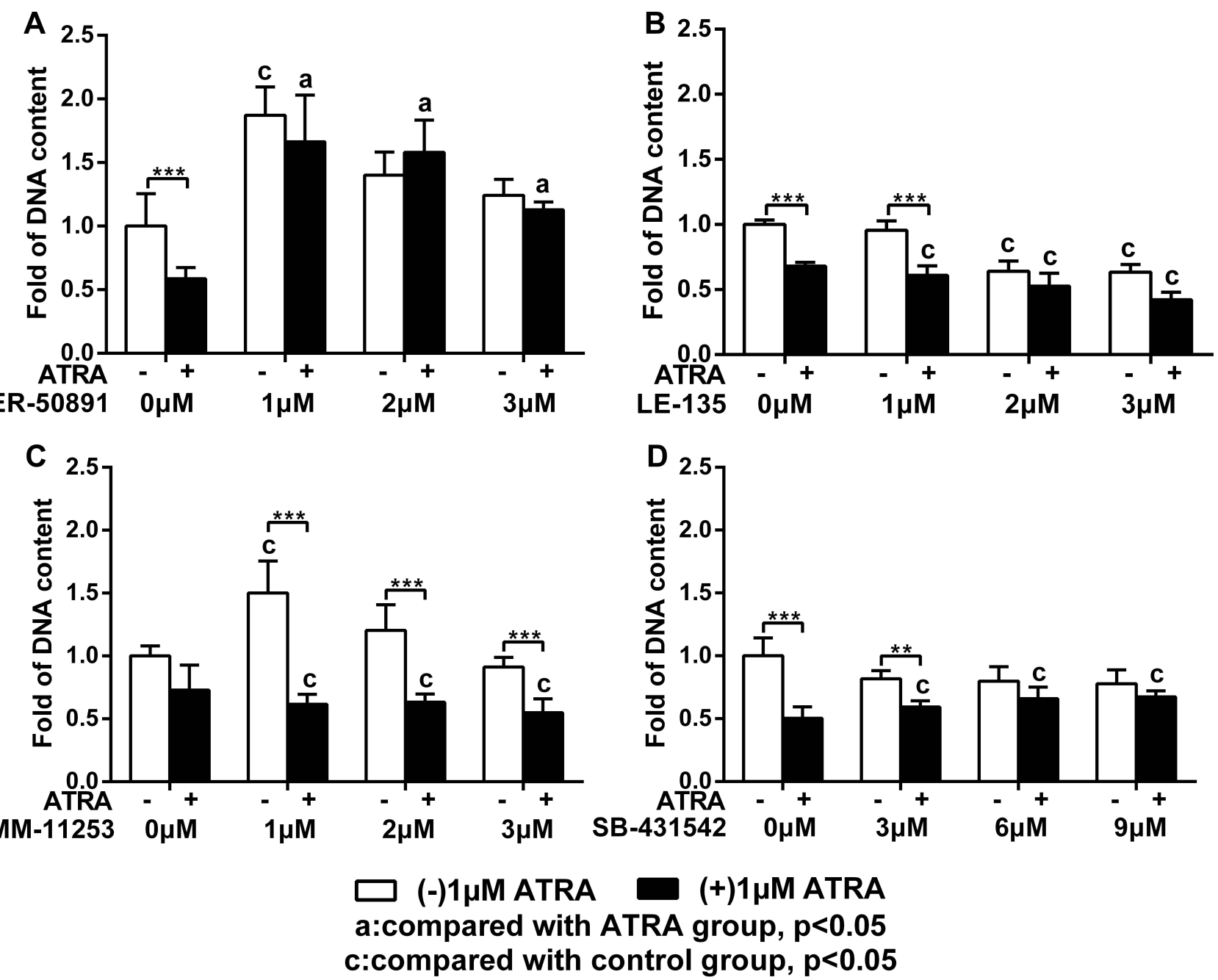

Figure 2 Fold changes of DNA content of murine calvarial pre-osteoblasts (MC3T3-EI cells) with or without I $\mu$ M ATRA in the presence or absence of different receptor antagonists. DNA contents of MC3T3-EI cells under different combinatory treatments of ATRA and RAR-alpha antagonist ER-5089I (A), RAR-beta antagonist LE-I35 (B), RAR-gamma antagonist MM-II 253 (C), and TGF-beta antagonist SB-43I542 (D). All data are presented as mean values together with the standard deviation. C: significant difference $(p<0.05)$ in comparison with the control group. a: significant difference $(p<0.05)$ in comparison with the ATRA (alone) group. ${ }^{* *} p<0.01$; $* * *<0.00$ I.

contrast, ATRA significantly decreased this parameter both in the absence (by 65.1\%) and presence (by 93.9\%) of BMP-2. ER-50891 alone did not significantly influence extracellular matrix mineralized nodules (Figure 5B). ER-50891 could rescue the formation of extracellular matrix mineralization to a level that was similar to the BMP-2 group (Figure 5B).

\section{Phosphorylated-Smad I/5 Was Not}

\section{a Target of Either ATRA or ER-5089I}

BMP-2 could significantly update the level of phosphorSmad1/5, while ATRA alone did not influence phosphor-Smad1/5 (Figure 6). Furthermore, ATRA could not significantly influence BMP-2 induced elevation of phosphor-Smad1/5. Similarly, ER-50891 did not influence phosphor-Smad1/5 both in the absence or presence of BMP-2 (Figure 6).

\section{Discussion}

ATRA has long been found as an important morphogen in regulating various biological events. ${ }^{40}$ Alcoholism, hypervitaminosis A or APL treatment can result in unphysiologically overdosed ATRA that might lead to osteoporosis by, at least partially, decreasing osteoblastogenesis. ${ }^{37,38}$ BMPs are a group of paramount growth factors for inducing physiological osteoblastogenesis and also widely used to promote bone regeneration in clinic. ${ }^{35,36}$ In our previous study, we demonstrate that ATRA can significantly antagonize the promoting effect of heterodimeric BMP2/7, a more potent form of BMPs, on osteoblastogenesis of preosteoblast cell line $\mathrm{e}^{38}$ 

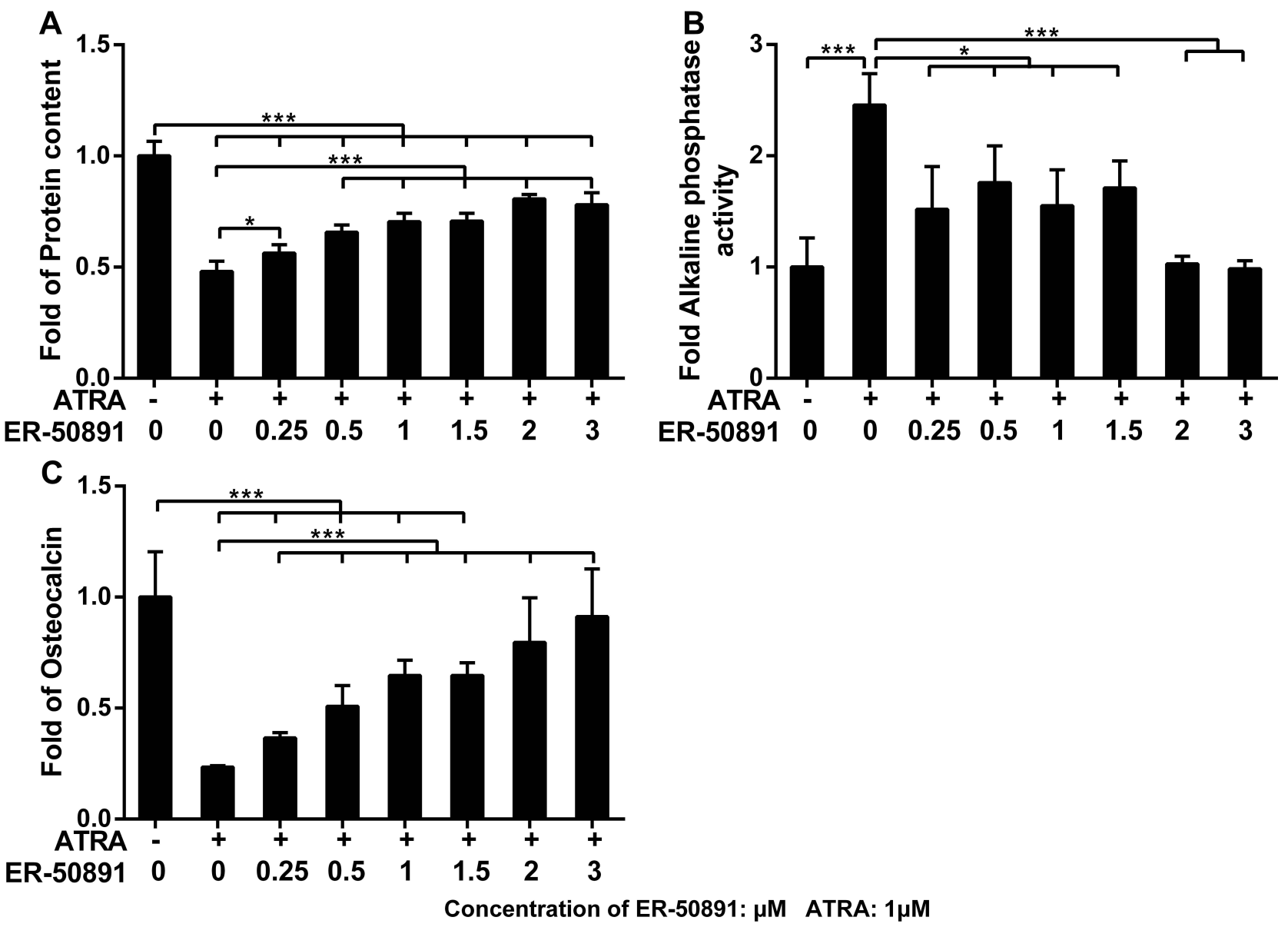

Figure 3 Fold changes of total protein content (A) ALP activity (B) and osteocalcin (C) of murine calvarial pre-osteoblasts (MC3T3-EI cells) treated with or without I $\mu$ M ATRA in presence or absence of different concentrations of RAR-alpha antagonist ER-5089I. All data are presented as mean values together with the standard deviation. $* p<0.05 ; * * * p<0.001$.

A

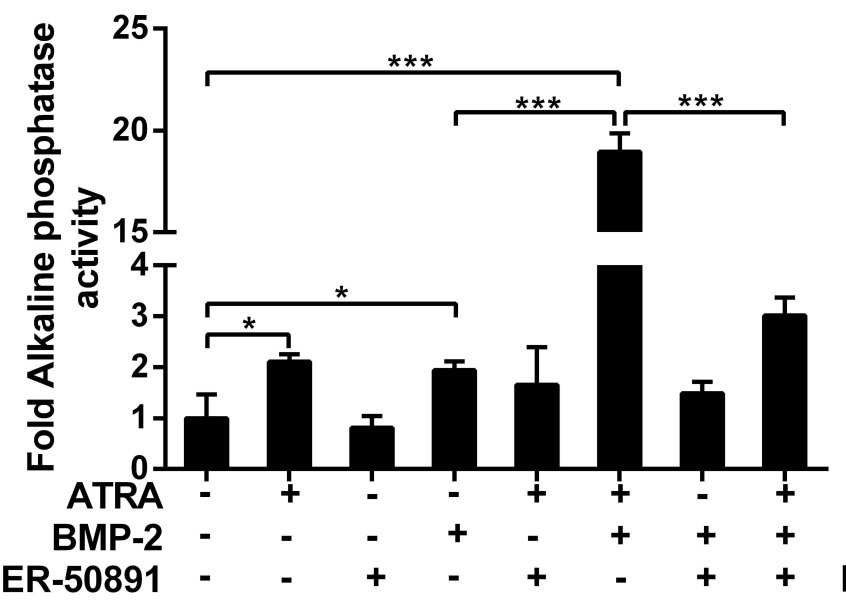

B

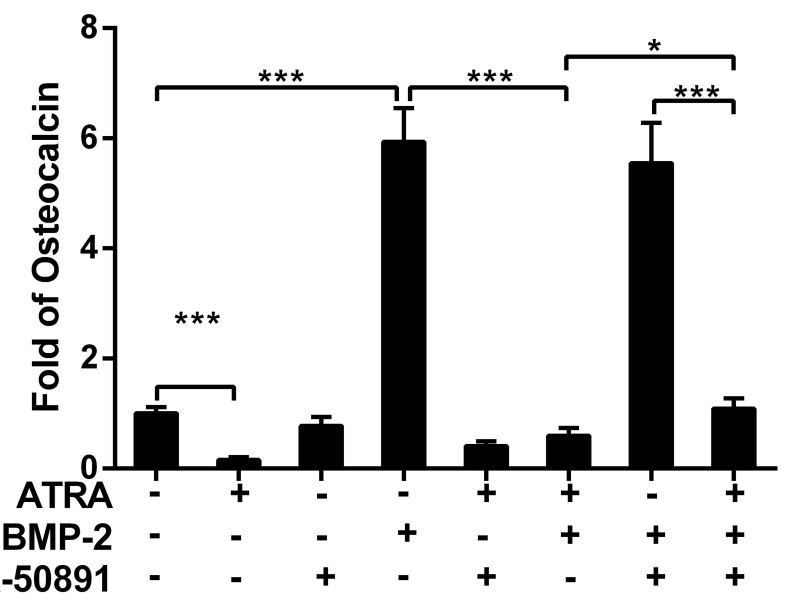

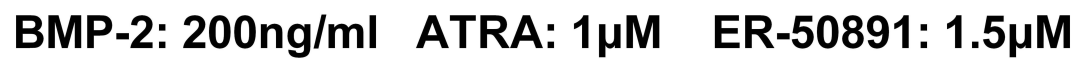

Figure 4 Fold changes of ALP activity (A) and osteocalcin (B) of murine calvarial pre-osteoblasts (MC3T3-EI cells) treated with or without I $\mu$ M ATRA and 200 ng/mL BMP-2 in presence or absence of I.5 $\mu$ M RAR-alpha antagonist ER-5089I. All data are presented as mean values together with the standard deviation. ${ }^{*}<0.05 ; * * * p<00$ I. 
A

Day19 mineralization
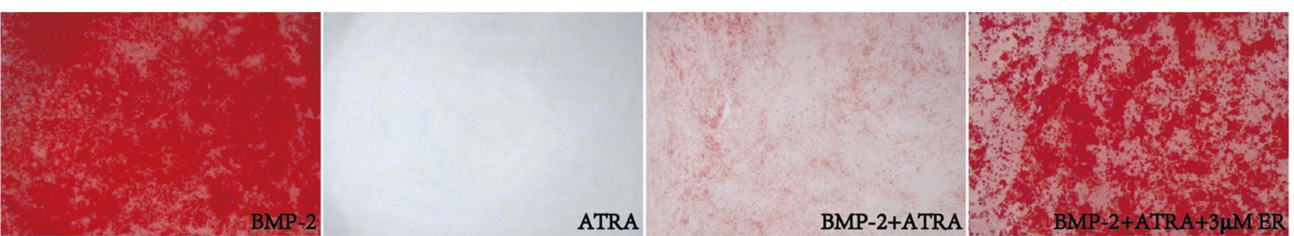

B Day19

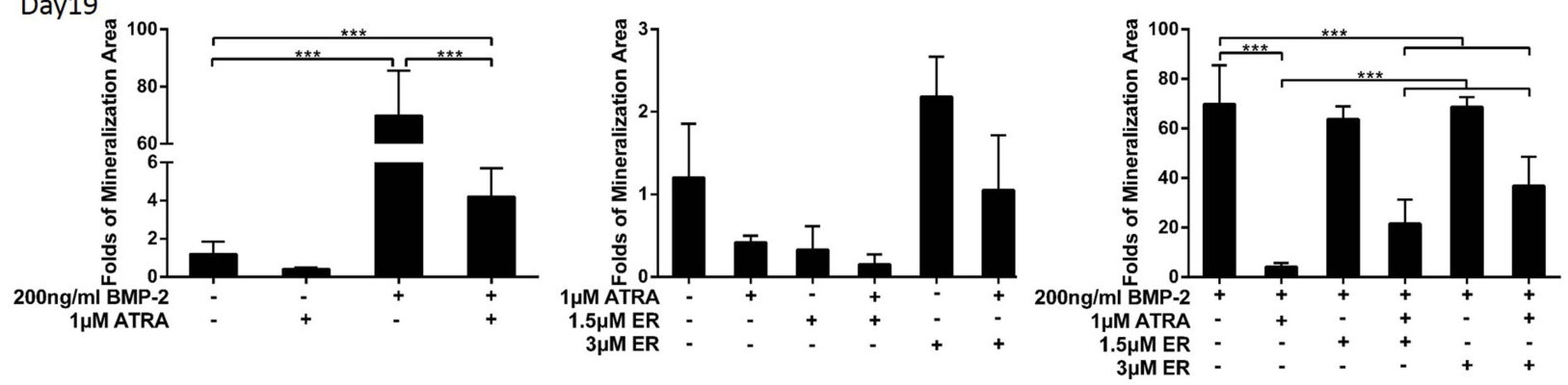

Figure 5 Alizarin red staining and Fold changes of mineralization area of murine calvarial pre-osteoblasts (MC3T3-EI cells) treated with or without I $\mu$ M ATRA in presence or absence of $200 \mathrm{ng} / \mathrm{mL}$ BMP-2 and different concentrations of RAR-alpha antagonist ER-5089I. Alizarin red staining (A), the mineralization area (B) of murine calvarial preosteoblasts (MC3T3-EI cells) under the different combinatory treatments of ATRA and BMP-2, in the absence and presence of RAR-alpha antagonist ER-5089I on Day I9. All data are presented as mean values together with the standard deviation. $* * * p<0.001$.

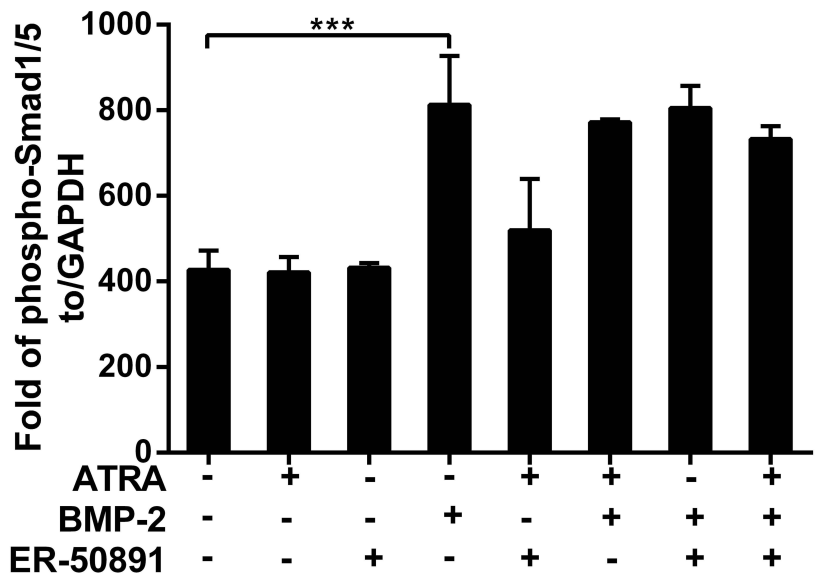

BMP2: 200ng/ml ATRA: $1 \mu M$ ER-50891: $1.5 \mu M$

Figure 6 Fold changes of phospho-SmadI/5 of murine calvarial pre-osteoblasts (MC3T3-El cells) treated with or without I $\mu$ M ATRA and $200 \mathrm{ng} / \mathrm{mL}$ BMP-2 in presence or absence of $1.5 \mu \mathrm{M}$. Fold change of phospho-Smad I/5 was normalized to GAPDH. All data are presented as mean values together with the standard deviation. $* * * p<0.001$.

and primary BMSCs. ${ }^{37}$ We further show that the inhibitive efficacy of ATRA is independent on the potencies of BMPs. ${ }^{39}$ However, it remains unveiled which receptor mediates the inhibitive effect of ATRA osteoblastogenesis in the absence or presence of BMP-2. In this study, we, for the first time, reported that the antagonist of RAR $\alpha$ but not RAR $\beta$ or RAR $\gamma$ or TGF- $\beta$ receptor significantly suppressed the inhibitive effects of ATRA and rescued osteoblastogenesis. This finding suggested that it was RAR $\alpha$ that mediated the inhibitive effects of ATRA.
ATRA exerts a large variations of effects through different RARs. RAR $\alpha$, also known as NR1B1, is encoded by the RARA gene and involved in many biological events, such as multicellular development, proliferation, differentiation and apoptosis. ${ }^{31}$ Enhanced expression of RARA could induce epithelial-to-mesenchymal transition. ${ }^{41}$ After decade's efforts, ATRA has been developed to aim at PMLRAR $\alpha$ and newly discovered NLS-RAR $\alpha$ oncoproteins as direct molecular targets based on epigenetic discoveries for APL involved HHEX and S100A10. ${ }^{42-46}$ RAR $\beta$ is encoded by RARB gene and has been considered to play a distinct role in the tumor growth and development. ${ }^{47}$ The RAR $\alpha$ and RAR $\beta$ can bind to RAREs to modulate target gene expression. RAR $\gamma$, encoded by RARG gene, is associated with skin, bone and teratogenic toxicity and is critical for hematopoiesis in modulating the balance between selfrenewal and differentiation of hematopoietic stem cells (HSCs). ${ }^{48}$ RAR signaling directly regulates osteoblastogenesis and adipogenesis. ${ }^{49,50}$ RAR $\gamma$ activity in mesenchymal stem cells plays a role in regulating endochondral bone. ${ }^{51}$ Green et al report that RAR $\gamma$ is a negative regulator of osteoclastogenesis in vivo and in vitro. ${ }^{49}$ However, hitherto, there are few publications reporting the involvement of different receptors in the ATRA's inhibitive effects of BMPinduced osteoblastogenesis.

Under the inhibitive effects of ATRA, mesenchymal cells or pre-osteoblasts are reported to exhibit a partially differentiated non-proliferating state. ${ }^{52}$ This character is 
symbolized by enhanced ALP activity (early osteoblastogenic marker) as well as suppressed cell proliferation/ viability, OCN expression and extracellular matrix mineralization. 6,37,38,52 However, hitherto, it remains unclear how ATRA leads to these phenomena. In this study, we showed that ATRA suppressed both total cell metabolic activity but also the proliferation (DNA content). ATRA resulted in a $30-50 \%$ reduction of DNA content and a $45-55 \%$ reduction of total cell metabolic activity. Therefore, the ATRA-induced reduction of total cell metabolic activity should be largely attributed to the inhibition of cell proliferation. It has long been established that retinoids inhibit cell proliferation by causing G1 arrest. $^{32,53}$ Such an effect of ATRA may be mediated via many mechanisms, among which endogenous TGF- $\beta$ s have been shown to mediate the anti-proliferative effect of ATRA. ${ }^{32-34}$ In this study, we showed that SB-431542, a specific inhibitor of TGF- $\beta$ type I receptor kinase, alone did not significantly influence cell viability or proliferation and could not antagonize the inhibitive effect of ATRA. This was complied with a previous publication that SB431542 showed no significant influence in the proliferation and osteogenic differentiation of pre-osteoblasts. ${ }^{54}$ These results suggested that endogenous TGF- $\beta$ s was not a mediator for ATRA's effect. Similar effect pattern was also found to MM-11253, RAR $\gamma$ antagonist. LE-135, antagonist of RAR $\beta$ alone even also suppressed total cell metabolic activity and proliferation, which suggested that endogenous RAR $\beta$-mediated signaling was a positive regulator for total cell metabolic activity and proliferation.

In contrast, RAR $\alpha$ antagonist alone significantly promoted the total cell metabolic activity and proliferation of preosteoblasts, which suggested that endogenous RAR $\alpha$ mediated signaling was a negative regulator for cell proliferation and total cell metabolic activity. Furthermore, under the presence of ATRA, RAR $\alpha$ antagonist also not only restored but also even promoted these two parameters to a level higher than the control, which suggested that RAR $\alpha$ mediated the suppressive effects of ATRA on cell proliferation and total cell metabolic activity. In our subsequent dose-dependent assays, RAR $\alpha$ antagonist significantly antagonized the effects of ATRA on both promoting ALP activity and also suppressing OCN expression and mineralization. These findings suggested that RAR $\alpha$ was one of the critical mediators for ATRA-induced inhibition of osteoblastogenesis.

In our previous publication, ${ }^{2}$ we reported that the heterodimeric BMP2/7 induced osteoblastogenesis at significantly lower optimal concentrations than homodimeric BMP2 BMP2/7 reached the maximal effect and was advantageous over BMP2 at $50 \mathrm{ng} / \mathrm{mL}$. Therefore, in our further publication, we adopted BMP2/7 to antagonize the inhibitory effects of ATRA on osteoblastogenesis ${ }^{37,38}$ However, in our most recent publication, we showed that although BMP2/7 was not advantageous over BMP2 in antagonizing the inhibitory effects of ATRA. ${ }^{39}$ Consequently, we adopted $200 \mathrm{ng} / \mathrm{mL}$ BMP2 in this study. The other reason to adopt BMP2 is that BMP2 combined with collagen membrane is an FDAapproved medical device and can be used in the clinic. Therefore, BMP2 is of more significance than BMP2/7 in clinical treatment. Hitherto, it remains largely unknown how ATRA suppresses BMP-induced osteoblastogenesis. One potential mechanism is that ATRA significantly decreased the signal duration of $\mathrm{p}-\mathrm{Smad} 1 / 5$ by promoting ubiquitination and proteasomal degradation. ${ }^{55}$ However, in this study, we showed that neither ATRA nor RAR $\alpha$ nor their combination significantly affected the level of $\mathrm{p}$-Smad $1 / 5$ induced by BMP-2. Recently, we have reported that ATRA could significantly inhibit the directly-upregulating genes (such as DLX5) of BMP signaling. ${ }^{39}$ Furthermore, we also found that the translocation of $\mathrm{p}$-Smad1/5 to nuclei was not significantly affected by ATRA (data not shown). All these findings suggested that ATRA inhibited BMP-2 signaling and its-induced osteoblastogenesis in translational level, such as the binding of $\mathrm{p}$-Smad $1 / 5$ to its targeting gene promoters. Further studies should be performed to investigate potential mechanisms.

In this study, we showed that ATRA enhanced ALP activity. This was consistent with previous studies that ATRA showed either no ${ }^{6,38}$ or promotive effect on ALP activity on various osteogenic cells, such as MC3T3-E1 preosteoblasts, rat UMR-201-10B preosteoblast cell line, C3H10T1/2 mesenchymal stem cells and primary mouse bone marrow stromal cells. ${ }^{37,56-58}$ Please note that the effect of ATRA on ALP gene expression is not through the classic osteogenic signaling pathways, such as promoting endogenous BMPs. Instead, this modulation of ATRA was shown to be highly dependent on a retinoid acid response element in the promoter of the ALP gene. ${ }^{58}$ Consequently, the ALP activity under the treatment of ATRA should be not considered as a conventional early osteogenic differential maker. In this study, our data showed that ATRA and BMP could synergistically enhance ALP activity to 18 folds of the control. The synergistic effect of ATRA and BMP is consistent with the previous reports from our and other researcher. ${ }^{37,59}$ However, in the later report, RAR $\gamma$ (but not 
RAR $\alpha$ or RAR $\beta$ )-dependent signaling can interact with $\mathrm{BMP} 2$ to regulate osteoblastic differentiation of $\mathrm{C} 2 \mathrm{C} 12$ cells. ${ }^{59}$ In contrast, in our results, the antagonist of RAR $\alpha$ but not RAR $\gamma$ suppressed the effects of ATRA. The inconsistency may be due to the different functions of RARs in different cell types. Please note that the promoting effect of ATRA on ALP gene expression is realized not by activating the classic osteogenic signaling pathways, such as enhancing the expression of endogenous BMPs. Instead, such an effect of ATRA is shown to be highly related to a retinoid acid response element (RARE) in the promoter of the ALP gene. ${ }^{58}$ Consequently, ALP promoted by ATRA or the combination of ATRA and BMP-2 might not be generally regarded as a marker of osteoblastogenic differentiation. Also, the statement that ATRA induces a partially differentiated status of cells might not be very precise. Although ATRA-induced ALP activity, it failed to enhance OCN expression and extracellular matrix mineralization. Thus, ATRA inhibited osteogenesis.

Interestingly, RAR $\alpha$ antagonist could only partially restore BMP-induced OCN expression to the same level as the control group. In fact, the osteocalcin level associated with the treatment of ATRA, ER and BMP-2 was significantly higher than that associated with the treatment of ATRA and BMP-2. However, such an effect of ER on OCN seemed not to be as dramatic as on ALP activity, which might suggest the involvement of other signaling mechanisms in the inhibition of OCN by ATRA. In this study, RAR $\alpha$ antagonist was selected largely basing on its antagonistic effect on the inhibited cell proliferation by ATRA rather than on osteogenic differentiation. There might be also a possibility that other RAR receptors than RAR $\alpha$ were involved in the inhibited osteogenic differentiation by ATRA. For example, RAR $\gamma$ was also shown to negatively influence BMP-2-induced bone formation. ${ }^{60}$ Consequently, further studies should be performed to reveal the inhibitory mechanisms of ATRA on osteocalcin expression. This might be one of the reasons that RAR $\alpha$ antagonist could not completely restore extracellular matrix mineralization. Consequently, in future studies, OCN may be used as a key parameter to check the effects of different receptor antagonists. Probably, a combination of two antagonists can completely nullify the inhibitory effect of ATRA on BMP-induced osteogenesis.

Previous cell studies show conflicting results regarding the role of retinoids on osteoblast differentiation. It is suggested that, to some extent, the effect of ATRA is dependent on the RA's concentration. ${ }^{61}$ Nanomolar
ATRA is reported to inhibit osteoblastogenic differentiation and functions, whereas micromolar ATRA stimulates osteoblastogenic differentiation. ${ }^{61}$ However, the dosedependent mechanisms are not sufficient to explain all the phenomena observed in the previous studies. ${ }^{39}$

One limitation in this study was that we only adopted a murine-derived preosteoblast cell line. Current results need to be further validated in primary cells. Besides, invivo study is also needed to show the interaction of ATRA and RAR-alpha antagonist in modulating in vivo BMP2induced new bone formation.

\section{Acknowledgment}

This study was supported by the funds of National Natural Science Foundation of China (81470724 and 81600844), Hebei Provincial Natural Science Foundation of China (H2017209238), Hebei Provincial Health Planning Commission Project Foundation of China (20180738 and 20191103), Zhejiang Provincial Natural Science Foundation of China (LY17H140010 and LQ18H140003), Zhejiang Provincial Department of Education (Y201636248), Science and Technology Department of Zhejiang Province (2017C33168).

\section{Disclosure}

The authors declare no conflict of interests.

\section{References}

1. Wakimoto M, Matsumura T, Ueno T, Mizukawa N, Yanagi Y, Iida S. Bone quality and quantity of the anterior maxillary trabecular bone in dental implant sites. Clin Oral Implants Res. 2012;23(11):1314-1319. doi:10.1111/clr.2012.23.issue-11

2. Zheng Y, Wu G, Zhao J, Wang L, Sun P, Gu Z. rhBMP2/7 heterodimer: an osteoblastogenesis inducer of not higher potency but lower effective concentration compared with rhBMP2 and rhBMP7 homodimers. Tissue Eng Part A. 2010;16(3):879-887.

3. Raggatt LJ, Partridge NC. Cellular and molecular mechanisms of bone remodeling. J Biol Chem. 2010;285(33):25103-25108.

4. Li J, Liu XY, Zuo B, Zhang L. The role of bone marrow microenvironment in governing the balance between osteoblastogenesis and adipogenesis. Aging Dis. 2016;7(4):514-525.

5. Aghebati-Maleki L, Dolati S, Zandi R, et al. Prospect of mesenchymal stem cells in therapy of osteoporosis: a review. $J$ Cell Physiol. 2019;234(6):8570-8578.

6. Wang A, Ding X, Sheng S, Yao Z. Retinoic acid inhibits osteogenic differentiation of rat bone marrow stromal cells. Biochem Biophys Res Commun. 2008;375(3):435-439.

7. Natesan S, Wrice NL, Christy RJ. Peroxisome proliferator-activated receptor-alpha agonist and all-trans retinoic acid induce epithelial differentiation of subcutaneous adipose-derived stem cells from debrided burn skin. J Cell Biochem. 2019;120(6):9213-9229.

8. Aouad P, Saikali M, Abdel-Samad R, et al. Antitumor activities of the synthetic retinoid ST1926 in two-dimensional and three-dimensional human breast cancer models. Anticancer Drugs. 2017;28(7):757-770. 
9. Smutna M, Priebojova J, Vecerkova J, Hilscherova K. Retinoid-like compounds produced by phytoplankton affect embryonic development of Xenopus laevis. Ecotoxicol Environ Saf. 2017;138:32-38.

10. Napoli JL. Physiological insights into all-trans-retinoic acid biosynthesis. Biochim Biophys Acta. 2012;1821(1):152-167. doi:10.1016/j.bbalip.2011.05.004

11. Centritto F, Paroni G, Bolis M, et al. Cellular and molecular determinants of all- trans retinoic acid sensitivity in breast cancer: luminal phenotype and RAR $\alpha$ expression. EMBO Mol Med. 2015;7 (7):950-972. doi:10.15252/emmm.201404670

12. Payne CM, Burke LP, Cavanagh B, O’Toole D, Cryan SA, Kelly HM. Evaluation of the immunomodulatory effects of all-trans retinoic acid solid lipid nanoparticles and human mesenchymal stem cells in an A549 epithelial cell line model. Pharm Res. 2019;36(4):50.

13. Kane MA, Folias AE, Wang C, Napoli JL. Ethanol elevates physiological all-trans-retinoic acid levels in select loci through altering retinoid metabolism in multiple loci: a potential mechanism of ethanol toxicity. FASEB J. 2010;24(3):823-832.

14. Peacock A, Leung J, Larney S, et al. Global statistics on alcohol, tobacco and illicit drug use: 2017 status report. Addiction. 2018;113:1905-1926.

15. Turner RT. Skeletal response to alcohol. Alcohol Clin Exp Res. 2000;24(11):1693-1701.

16. Schnitzler CM, Mesquita JM, Shires R. Cortical and trabecular bone microarchitecture and turnover in alcohol-induced chronic pancreatitis: a histomorphometric study. J Bone Miner Metab. 2010;28(4):456-467.

17. Backo H, Love J. Alcohol-induced osteopenia among in-custody and homicide deaths from the harris county institute of forensic sciences. J Forensic Sci. 2013;58(4):1055-1060.

18. Ulhoi MP, Meldgaard K, Steiniche T, Odgaard A, Vesterby A. Chronic alcohol abuse leads to low bone mass with no general loss of bone structure or bone mechanical strength. J Forensic Sci. 2017;62:131-136.

19. Kanis JA, Johansson H, Johnell O, et al. Alcohol intake as a risk factor for fracture. Osteoporosis Int. 2005;16(7):737-742.

20. Michael AR, Bengtson JD. Chronic alcoholism and bone remodeling processes: caveats and considerations for the forensic anthropologist J Forensic Leg Med. 2015;38:87-92.

21. Gonzalez-Reimers E, Quintero-Platt G, Rodriguez-Rodriguez E, Martinez-Riera A, Alvisa-Negrin J, Santolaria-Fernandez F. Bone changes in alcoholic liver disease. World J Hepatol. 2015;7(9):1258-1264.

22. Camilli JA, da Cunha MR, Bertran CA, Kawachi EY. Subperiosteal hydroxyapatite implants in rats submitted to ethanol ingestion. Arch Oral Biol. 2004;49(9):747-753.

23. Lima CC, Silva TD, Santos L, et al. Effects of ethanol on the osteogenesis around porous hydroxyapatite implants. Braz J Biol. 2011;71(1):115-119.

24. Trevisiol CH, Turner RT, Pfaff JE, et al. Impaired osteoinduction in a rat model for chronic alcohol abuse. Bone. 2007;41(2):175-180.

25. Carvalho IC, Martinelli Cda S, Milhan NV, et al. Prenatal alcohol exposure reduces mandibular calcium and phosphorus concentrations in newborn rats. $J$ Oral Sci. 2016;58(3):439-444.

26. Lo-Coco F, Avvisati G, Vignetti M, et al. Retinoic acid and arsenic trioxide for acute promyelocytic leukemia. $N$ Engl J Med. 2013;369 (2):111-121.

27. Smith MA, Adamson PC, Balis FM, et al. Phase I and pharmacokinetic evaluation of all-trans-retinoic acid in pediatric patients with cancer. J Clin Oncol. 1992;10(11):1666-1673.

28. Muindi JR, Frankel SR, Huselton C, et al. Clinical pharmacology of oral all-trans retinoic acid in patients with acute promyelocytic leukemia. Cancer Res. 1992;52(8):2138-2142.

29. Saneshige S, Mano H, Tezuka K, et al. Retinoic acid directly stimulates osteoclastic bone resorption and gene expression of cathepsin $\mathrm{K}$ / OC-2. Biochem J. 1995;309(Pt 3):721-724.

30. Tomita A, Kiyoi H, Naoe T. Mechanisms of action and resistance to all-trans retinoic acid (ATRA) and arsenic trioxide (As2O 3) in acute promyelocytic leukemia. Int J Hematol. 2013;97(6):717-725.
31. Benbrook DM, Chambon P, Rochette-Egly C, Asson-Batres MA. History of retinoic acid receptors. Subcell Biochem. 2014;70:1-20.

32. Yang L, Ostrowski J, Reczek P, Brown P. The retinoic acid receptor antagonist, BMS453, inhibits normal breast cell growth by inducing active TGFbeta and causing cell cycle arrest. Oncogene. 2001;20 (55):8025-8035.

33. Nunes I, Kojima S, Rifkin DB. Effects of endogenously activated transforming growth factor-beta on growth and differentiation of retinoic acid-treated HL-60 cells. Cancer Res. 1996;56(3):495-499.

34. Fernandez-Calotti P, Pastor-Anglada M. All-trans-retinoic acid promotes trafficking of human concentrative nucleoside transporter-3 (hCNT3) to the plasma membrane by a TGF-beta1-mediated mechanism. J Biol Chem. 2010;285(18):13589-13598.

35. Bessa PC, Casal M, Reis RL. Bone morphogenetic proteins in tissue engineering: the road from laboratory to clinic, part II (BMP delivery). J Tissue Eng Regen Med. 2008;2(2-3):81-96.

36. Bessa PC, Casal M, Reis RL. Bone morphogenetic proteins in tissue engineering: the road from the laboratory to the clinic, part I (basic concepts). J Tissue Eng Regen Med. 2008;2(1):1-13.

37. Bi W, Gu Z, Zheng Y, Wang L, Guo J, Wu G. Antagonistic and synergistic effects of bone morphogenetic protein $2 / 7$ and all-trans retinoic acid on the osteogenic differentiation of rat bone marrow stromal cells. Dev Growth Differ. 2013;55(9):744-754.

38. Bi W, Gu Z, Zheng Y, Zhang X, Guo J, Wu G. Heterodimeric BMP-2/7 antagonizes the inhibition of all-trans retinoic acid and promotes the osteoblastogenesis. PLoS One. 2013;8(10):e78198.

39. Liu Y, Ma X, Guo J, et al. All-trans retinoic acid can antagonize osteoblastogenesis induced by different BMPs irrespective of their dimerization types and dose-efficiencies. Drug Des Devel Ther. 2018;12:3419-3430.

40. Kam RK, Deng Y, Chen Y, Zhao H. Retinoic acid synthesis and functions in early embryonic development. Cell Biosci. 2012;2(1):11.

41. Doi A, Ishikawa K, Shibata N, et al. Enhanced expression of retinoic acid receptor alpha (RARA) induces epithelial-to-mesenchymal transition and disruption of mammary acinar structures. Mol Oncol. 2015;9(2):355-364.

42. Diverio D, Lo Coco F, D'Adamo F, et al. Identification of DNA rearrangements at the retinoic acid receptor-alpha (RAR-alpha) locus in all patients with acute promyelocytic leukemia (APL) and mapping of APL breakpoints within the RAR-alpha second intron. Italian cooperative study group "GIMEMA”. Blood. 1992;79(12):3331-3336.

43. Nitto T, Sawaki K. Molecular mechanisms of the antileukemia activities of retinoid and arsenic. J Pharmacol Sci. 2014;126(3):179-185.

44. O'Connell PA, Madureira PA, Berman JN, Liwski RS, Waisman DM. Regulation of S100A10 by the PML-RAR-alpha oncoprotein. Blood. 2011;117(15):4095-4105.

45. Saulle E, Petronelli A, Pelosi E, et al. PML-RAR alpha induces the downmodulation of HHEX: a key event responsible for the induction of an angiogenetic response. J Hematol Oncol. 2016;9:33.

46. Valiuliene G, Treigyte G, Savickiene J, et al. Histone modifications patterns in tissues and tumours from acute promyelocytic leukemia xenograft model in response to combined epigenetic therapy. Biomed Pharmacother. 2016;79:62-70.

47. Fang C, Jian ZY, Shen XF, Wei XM, Yu GZ, Zeng XT. Promoter methylation of the retinoic acid receptor beta2 (RARbeta2) is associated with increased risk of breast cancer: a PRISMA compliant meta-analysis. PLoS One. 2015;10(10):e0140329.

48. Purton LE, Dworkin S, Olsen GH, et al. RARgamma is critical for maintaining a balance between hematopoietic stem cell self-renewal and differentiation. J Exp Med. 2006;203(5):1283-1293.

49. Green AC, Poulton IJ, Vrahnas C, et al. RARgamma is a negative regulator of osteoclastogenesis. J Steroid Biochem Mol Biol. 2015;150:46-53.

50. Green AC, Kocovski P, Jovic T, et al. Retinoic acid receptor signalling directly regulates osteoblast and adipocyte differentiation from mesenchymal progenitor cells. Exp Cell Res. 2017;350(1):284-297. 
51. Green AC, Rudolph-Stringer V, Straszkowski L, et al. Retinoic acid receptor gamma activity in mesenchymal stem cells regulates endochondral bone, angiogenesis, and B lymphopoiesis. J Bone Miner Res. 2018;33(12):2202-2213.

52. Kitching R, Qi S, Li V, Raouf A, Vary CP, Seth A. Coordinate gene expression patterns during osteoblast maturation and retinoic acid treatment of MC3T3-E1 cells. J Bone Miner Metab. 2002;20 (5):269-280.

53. Yang L, Munoz-Medellin D, Kim HT, Ostrowski J, Reczek P, Brown PH. Retinoic acid receptor antagonist BMS453 inhibits the growth of normal and malignant breast cells without activating RAR-dependent gene expression. Breast Cancer Res Treat. 1999;56 (3):277-291

54. Li Y, Wang J, Ren F, et al. Lactoferrin promotes osteogenesis through TGF-beta receptor II binding in osteoblasts and activation of canonical TGF-beta signaling in MC3T3-E1 cells and C57BL/6J mice. J Nutr. 2018;148(8):1285-1292.

55. Sheng N, Xie Z, Wang C, et al. Retinoic acid regulates bone morphogenic protein signal duration by promoting the degradation of phosphorylated Smad1. Proc Natl Acad Sci U S A. 2010;107 (44):18886-18891.
56. Choong PF, Martin TJ, Ng KW. Effects of ascorbic acid, calcitriol, and retinoic acid on the differentiation of preosteoblasts. J Orthop Res. 1993;11(5):638-647.

57. Gazit D, Ebner R, Kahn AJ, Derynck R. Modulation of expression and cell surface binding of members of the transforming growth factor-beta superfamily during retinoic acid-induced osteoblastic differentiation of multipotential mesenchymal cells. Mol Endocrinol. 1993;7(2):189-198.

58. Hisada K, Hata K, Ichida F, et al. Retinoic acid regulates commitment of undifferentiated mesenchymal stem cells into osteoblasts and adipocytes. J Bone Miner Metab. 2013;31(1):53-63.

59. Karakida T, Yui R, Suzuki T, Fukae M, Oida S. Retinoic acid receptor gamma-dependent signaling cooperates with BMP2 to induce osteoblastic differentiation of C2C12 cells. Connect Tissue Res. 2011;52 (5):365-372.

60. Uchibe K, Son J, Larmour C, Pacifici M, Enomoto-Iwamoto M, Iwamoto M. Genetic and pharmacological inhibition of retinoic acid receptor gamma function promotes endochondral bone formation. J Orthop Res. 2017;35(5):1096-1105.

61. Henning P, Conaway HH, Lerner UH. Retinoid receptors in bone and their role in bone remodeling. Front Endocrinol (Lausanne). 2015;6:31.

\section{Publish your work in this journal}

Drug Design, Development and Therapy is an international, peerreviewed open-access journal that spans the spectrum of drug design and development through to clinical applications. Clinical outcomes, patient safety, and programs for the development and effective, safe, and sustained use of medicines are a feature of the journal, which has also been accepted for indexing on PubMed Central. The manuscrip management system is completely online and includes a very quick and fair peer-review system, which is all easy to use. Visit http://www. dovepress.com/testimonials.php to read real quotes from published authors. 